\title{
Resource Discovery in High-Volume Internet of Things: Systematic Research
}

\author{
Mohd Tamizan Abu Bakar, Azrul Amri Jamal, Elissa Nadia Madi
}

\begin{abstract}
Currently, several issues in Internet of Things (IoT) resource discovery is still being analyzed and verified. This paper offers a comparison with systematic literature and solution perspective on the resources discovery issues in the high-volume IoT environment. The purpose is to recommend a clear review on how the resources could be maximize or improve properly within different IoT solutions. We compared and reviewed all IoT resource discovery literature and solutions, categorizing a final collection of 19 related papers and then produce summarisation in three different tables. Based on the outputs, we are able to provide an overview of the IoT resource discovery categories, main areas of the research, IoT platforms, simulation tools and recommended solutions. Finally, we hope this review can provides insight result for every IoT sources, propose appropriate techniques or methods that can help to ensure the IoT resource discovery becomes most effective in the future..
\end{abstract}

Keywords: High-volume Industrial Internet of Things (IIoT), IoT, Internet of Things (IoT), LoRa, NS-3 simulator, resource discovery..

\section{INTRODUCTION}

IoT ecosystems exist all around us, it has become a global phenomenon. IoT is currently the fastest expanding technology and has become more important in the recent years. Alongside the development of Wireless Sensor Networks (WSN), cellular lines, WiFi, LiFi, Radio Frequency Identification (RFID), sensors, Global Positioning System Satellite Networks (GPS), Long-Range Wireless (LoRa) and other related technologies, IoT has been widely applied in many applications successfully and plays a pivotal role in many transportation and monitoring systems [1]. IoT helps individuals connect things to improve quality of life. It also helps organisations and industries improve resource management in order to become more efficient and cost effective. The IoT is helping industries, public and private sector organisations increase operational efficiency. This act boosts the emergence of high-volume IoT, also known as Industrial Internet of Things (IIoT). Researchers in academia and industries are now increasingly deploying brand new IoT solutions to the market. Several innovation ideas that supports the IoT ecosystems appeared such as the narrow-band IoT (NB-IoT) and LoRa [2], [3].

The rapid increase in IoT system implementation has led to issues such as network latency, privacy, public acceptance, and, security [4]. IoT challenges including methods to combine millions of IoT devices developed by different vendors and unceasing number of new IoT devices needed to be integrated into existing network infrastructure [5]. As summarised in Fig. 1, IoT can be divided into 2 main categories namely Industrial IoT and Massive IoT [1], [13], [22], [23]. Nevertheless, IIoT (or high-volume IoT) and massive IoT still have their own unresolved problems such as in regards to security, data privacy, service management, data abstraction, programmability, optimisation metrics, and energy sources [6]. These are due to the inadequacy of IoT's maturity and the constraints on existing appliances. Today, the problems of delay and latency while exploring sources in a large-scale or high-volume IoT environment are considered a common problem. Three contributing factors to latency in IoT are clustering process, server utilisation, and application [7]. Resource discovery techniques in highvolume IoT network environments are complicated when engaging with a large number of devices or occur in IIoT. To meet the demand for this new network technology, many researchers are still focus on the increasing performance for random access processes on uplink (UL) lines [8]-[10]. Nonetheless, that solution is just a suggestion and it is still in the research stage. This paper reviews clearly the unresolved issues related to resources discovery within the IoT environment using a suitable simulation tool.

\section{IOT LORA-BASED PLATFORM}

IoT is the fastest growing technology due to the active development of numerous communication technologies such as RFID, WSN, LoRa, Wi-Fi, ZigBee, NFC, BLE, LTE, and SigFox [3]. IoT devices are entering the market using its own data transfer techniques. Each of these communication technologies has its own unique and distinct advantages. Some researcher ideas have emerged including the NB-IoT and also the latest high-speed mobile IoTs such as 4G LTE and 5G. Nevertheless, this rapid increase also introduces new challenges such as IoT latency and security especially when it involves IIoT [7]. Challenges to IoT can be categorised into three different situations such as the integration of IoT devices from different vendors each using custom applications; the integration of new IoT devices to existing network infrastructures; and the security of new IoT devices with varying levels of security configuration.

Revised Manuscript Received on September 14, 2019.

Mohd Tamizan Abu Bakar, Faculty of Computer, Media and Technology, TATI University College, Jalan Panchor, Teluk Kalong, Kemaman, Terengganu, 24000, Malaysia.

Azrul Amri Jamal, Faculty of Informatics and Computing, Universiti Sultan Zainal Abidin, Besut Campus, Besut, Terengganu 22200, Malaysia.

Elissa Nadia Madi, Faculty of Informatics and Computing, Universiti Sultan Zainal Abidin, Besut Campus, Besut, Terengganu 22200, Malaysia. 


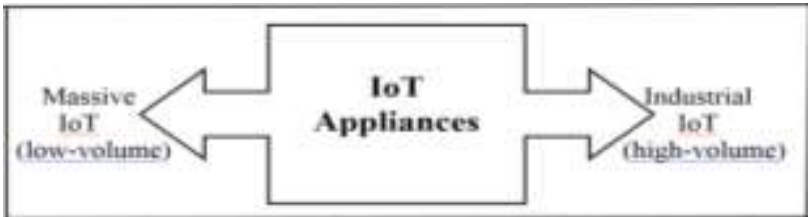

Fig. 1: Categories of basic IoT [1], [13], [22], [23]

The rapid IoT growth also introduced new challenges including platform selection matters. IoT platforms provide various capabilities in all environments. Various platforms in network communication will provide several options for IIoT to customise existing technologies with their features. We look forward to LoRa as the best option to support IIoT as some of the factors proposed by previous researchers [11], [12].

Selecting the right IoT platform is the most challenging process for a company. This challenge includes how to communicate thousands of IoT devices from different vendors and how to integrate new IoT appliances into current network infrastructure in the future. Choosing an appropriate IoT platform for a given field of application is a challenge task when selection from the mess of different platforms for massive IoT [13]. LoRa communication has received much attention and cooperation from researchers and industries for supporting IoT using embedded machine learning and sensors [14], [15]. Due to its capability of supporting long range communication especially for highvolume IoT, LoRa can fill the gap between mobility and low power [16]. Several parameters are listed here to support the selection of LoRa as a platform for large-scale network IoT. Table 1 shows the selected parameters based on the suitable implementations by the difference LoRa researchers

Table 1: Performance of LoRa in different parameters

\begin{tabular}{|c|c|c|c|}
\hline No. & LoRa Parameters & Implementation & Result \\
\hline 1 & Performance [17] & $\begin{array}{l}\text { Monitored LoRa performance } \\
\text { using } 5 \text { parameters - carrier } \\
\text { frequency, spreading factor, } \\
\text { transmission power, bandwidth } \\
\text { and coding rate. } \\
\text { Indoor experiment. } \\
\text { Outdoor experiment. }\end{array}$ & $\begin{array}{l}\text { The result can reduce the size of the package, thus reducing the number } \\
\text { of redelivery and lost packages. } \\
\text { Results show that communication can be reached without problems and } \\
\text { packet loss is minimum. }\end{array}$ \\
\hline 2 & Security [18] & $\begin{array}{l}\text { Proposed a new LoRa structure } \\
\text { as the trusted third party. The } \\
\text { latest version LoRa v1.1 } \\
\text { addressed many security } \\
\text { problems previously reported. } \\
\text { Their work introduced the main } \\
\text { features of LoRa and potential } \\
\text { threats to the protocol security. }\end{array}$ & $\begin{array}{l}\text { The security of LoRa-based systems will evidently depend on } \\
\text { implementation aspects. } \\
\text { They provide a solution of security threats involved like RF Jamming } \\
\text { Attack, Replay Attack, Beacon Synchronisation Attack, Network Traffic } \\
\text { Analysis, and Man In The Middle Attack. }\end{array}$ \\
\hline 3 & Operation [19] & $\begin{array}{l}\text { Operate LoRa using chirp } \\
\text { spread spectrum with different } \\
\text { Spreading Factors to trade of } \\
\text { data rate for extra range. These } \\
\text { spreading factors specify the } \\
\text { chirp rate. }\end{array}$ & $\begin{array}{l}\text { This leads to higher delivery rates but shorter delivery times, but } \\
\text { increases service rates. }\end{array}$ \\
\hline 4 & New Idea [19] & $\begin{array}{l}\text { Proposed a new LoRa module } \\
\text { in NS-3 simulator. }\end{array}$ & $\begin{array}{l}\text { Make easy integration of new algorithms on the server side to investigate } \\
\text { new protocols and the effect of different parameters on the network. }\end{array}$ \\
\hline 5 & Reliability [17] & $\begin{array}{l}\text { Partitioned whole packet based } \\
\text { on the LoRa frame structure to } \\
\text { investigate the circumstances } \\
\text { of collisions and frame loss. }\end{array}$ & $\begin{array}{l}\text { The result shows that the frame is still able to survive even in the } \\
\text { collision as long as the last six symbols of the preamble and the title do } \\
\text { not collide. }\end{array}$ \\
\hline 6 & $\begin{array}{l}\text { Scalability [17], } \\
{[20]}\end{array}$ & $\begin{array}{l}\text { Measure LoRa scalability to } \\
\text { keep all nodes active at the } \\
\text { same time. }\end{array}$ & $\begin{array}{l}\text { An analysis of scalability for LPWA technology has been made for a } \\
\text { real sensing environment. The process of exploring the recognition of } \\
\text { this activity by using LoRa sensors. }\end{array}$ \\
\hline 7 & $\begin{array}{l}\text { Frame Collision } \\
{[20]}\end{array}$ & 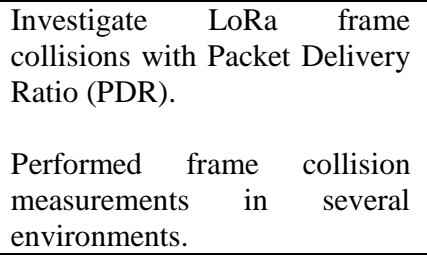 & $\begin{array}{l}\text { Results reveal an improved PDR in single gateway scenario. The } \\
\text { stronger frames could be decoded properly, if not the weaker frames } \\
\text { could not reach the more distant gateways. The higher PDR levels was } \\
\text { contributed by the closer gateway. } \\
\text { Adding more gateways can improve DER percentage because results } \\
\text { show that DER mostly depends on the distance between the devices and } \\
\text { gateways for a single gateway. }\end{array}$ \\
\hline 8 & $\begin{array}{c}\text { Physical Layer } \\
\text { [21] }\end{array}$ & $\begin{array}{l}\text { Analyse LoRa physical layer } \\
\text { performance in several IoT use } \\
\text { cases. }\end{array}$ & $\begin{array}{l}\text { LoRa have several advantages like long range, multipath resistance, } \\
\text { robustness, low power consumption, forward error correction, and so on. } \\
\text { Physical layer parameters measured in LoRa include spreading factor, } \\
\text { bandwidth, transmission power, and code rate. }\end{array}$ \\
\hline
\end{tabular}




\section{EVOUTION OF HIGH-VOLUME IOT}

IoT networks are a set of connections between large networks of large dimension devices. It does not merely refer to connecting equipment, but also refers to the overall scale for the level of separate traffic breakdown that must be provided primarily involving the main connecting route. Wireless technology is the most critical requirement to support the current trends generated by current IoT equipment [3]. The statements above have highlighted the importance of LoRa to support IIoT. Massive IoT arises with the emergence of various IoT appliances from various independence platforms. The trends of emergence is towards without emphasizing the speed of data access that should be available on IoT devices, while IIoT implies the use of critical IoT equipment in the process of high-volume data accessing by involving specialised IoT appliances especially in mega industry field, healthcare, smart city, and critical disaster area [5], [30], [31].

To achieve the right objectives of the IIoT resources discovery, a series of experiments are needed to investigate the delay or latency of data transfers in the IoT environment, especially in high scale networks. It covers the standard data transfer and data parsing in the cloud. For long term research, three IoT domains must be identified including infrastructure, nomadic users, and digital economy. A model for flexible IoT system was proposed to achieve the understanding of studies in fault-tolerant (robustness), dependability, reliability, and availability of the system. This wireless technology requires a new paradigm to be improved in terms of placement and random resource access, to solve the problems such as bottlenecks that eventually become a barrier to wireless technology to support the development of this high-volume IoT. Generally, challenges in IIoT can be divided into three types [24]:

i) IIoT System Resistance

ii) IIoT System Optimisation

iii) IIoT Resource Management

Industry can look forward towards the involvement of IIoT's resource management in the development of ubiquitous computing. Although research-related ubiquitous computing systems have long been implemented, there are still many questions about how to incorporate the privacy controls that can be used in specific systems [25]. The process of discovering resources in ubiquitous computing is a new dimension where information processing is associated with every activity or object found by involving connecting electronic devices, including embedding microprocessors to convey information. Ubiquitous computing focuses on improving the results by removing the computing technologies latency, complexity and increasing efficiency the usage of computing for different daily activities in critical IoT environments such as smart city, e-health, and intelligent vehicles. IoT components that use ubiquitous computing have several interesting features included constant availability and completely connected [6], [17], [25].

Many new IoT devices, applications, protocols, standard, architectures and models are being developed. For example, the idea of fog computing was introduced as a bridge between IoT and the cloud [26]. Fog Computing is a new era of cloud computing paradigms that bring cloud services to the edge of the network to make IoT devices more controllable. [27]-[31]. To simplify the issue of ubiquitous computing in the study area, we should highlight the problem background in Fig. 2. The ability of IIoT appliances to support high embeddedness and mobility are critical features for ubiquitous computing.

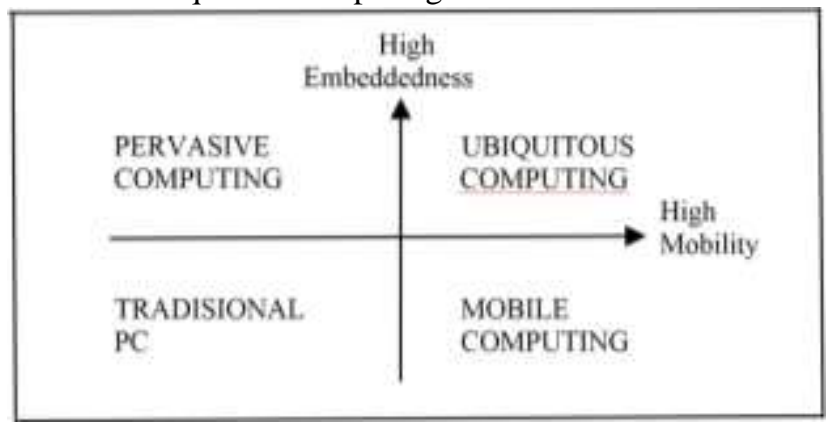

Fig. 2: Relationship of computing system categories

[17], [25]

\section{RESOURCES DSICOVERY FOR IIOT}

Data can be words in a book, contents of a spreadsheet, photos, files, or streams of measurements sent by devices Data growth can be categorised as linear and exponential. Data can no longer be stored on a few machines or processed with just one tool. After it has been gathered, prepared, analysed, and presented in a usable format, data needs to be changed from its raw format into usable information. Together with the advancement of IoT, data has become either more vast, faster, or more complex that it would be impossible to store, process, and analyse using traditional data storage and analytics applications.

As well as data usage in the other field, IoT data are collected from wired or wireless networks of smart things like devices, sensors, and services. However, IoT data more concerns in large volume and different forms may require more advanced analytic tools for different types of data, and it is frequently streaming in real-time or nearly real-time [32]. So, the IoT data from the connected things are continuously generated as data streams because the IoT data either structured or unstructured must be organised in real time.

Commonly data needs cleaning, converting, and manipulating before proceeding to data analysis. However, on searching the data in IoT, there are three significant activities including indexing, discovery, and ranking solutions [33]. In terms of resource discovery, it refers to crawling, finding, and allowing IoT resources to be discovered automatically or manually. Indexing means sorting the IoT data to allow fast accessing to their resources, including retrieval and searching for the data. Discovery means to find and discover data resources that can answer a requested query given the key search attributes. While ranking means prioritising the IoT resources based on several criteria given either functional or non-functional. The focus of our study here is narrow and 
only to the resource discovery for IoT.

Resource discovery is about finding and accessing specific sources to get the requested data to analyse it. Highvolume IoT resource discovery brings another dimension of challenges to crawl, find, access and analysis of online data from different sources, while other data are continuously published. The accessing process is based on the requested query to search for sources, patterns, or data events. Collaborative services are used on data obtained from various sources to make analysis and give intelligent results. Resource discovery could be limited by a time interval in the processing of data for disaster monitoring [34]. Furthermore, to support loT application to provide rich ubiquitous connectivity, the elements of '6A' connectivity are required (Anytime, Anyplace, Any service, Anything, Anyone, and Any path) [35].

In the meantime, in [35] researcher in the resource management field said, resource discovery within the mobile IoT network can be classified into different scenarios as follows:

i) Time Synchronised Protocols Approach

ii) Deterministic Approach

iii) Colocation based Approaches

iv) Fully Distributed Opportunistic Approach

v) Learning Based Approach (Context-Aware Resource Discovery Approach)

However, not all resource discovery methods are acceptable if they are not corrected using special techniques or extra elements. For example, the Breadth-First Search Technique (BFS) is the earliest source discovery mechanisms derived from mathematical formulation models and is still used for resource discovery in unstructured P2P networks. According to [36] by implementing the alpha multipliers, the BFS was able to increase the combined efficiency rate. This method shows that the performances for the most discovery methods can be increased if any suitable element adds to the discovery processes. Categories of resource discovery techniques in grid computing systems as follows:
i) Centralised
ii) Decentralised
iii) Peer-to-Peer (Unstructured / Structured / Super-
Peer / Hybrid)
iv) Hierarchical
v) Agent Based

The IoT and IIoT applications progress on today can be classified into Society, Industry and Environment domains [37]. Refer to the idea of (Pattar, Buyya, Venugopal, Iyengar, \& Patnaik, 2018), the searching techniques in any IoT domains are variety and difference such as event-based search, location-based search, time-related search, contentbased search, spatiotemporal-based search, context-based search, real-time search, and interactive user search. In short, searching systems will operate based on classifications as below:
i) Text-based Approach
ii) Metadata-based Approach
iii) Ontology-based Approach

In summary, the techniques discussed in the field of IoT resource discovery has been refined to clarify their performance for future use. We list in Table 2 each of the related techniques and compare their main advantage and main disadvantage as a guide for future researchers

Table 2: Main advantage and disadvantage for each discovery techniques

\begin{tabular}{|c|c|c|c|}
\hline No. & IoT Discovery Techniques & Main Advantage & Main Disadvantage \\
\hline 1 & ZebraNet [35] & $\begin{array}{l}\text { Data collection with energy } \\
\text { efficiency. }\end{array}$ & Low performance with network changes. \\
\hline 2 & $\begin{array}{c}\text { Recursive Binary Tree } \\
\text { Partitioning (RBTP) [35] }\end{array}$ & Battery saving. & $\begin{array}{l}\text { Node does not consider the arrival } \\
\text { pattern of neighbours. }\end{array}$ \\
\hline 3 & Wake-Up Scheduling [35] & $\begin{array}{c}\text { Minimise forward propagation } \\
\text { delay. }\end{array}$ & Waiting for the neighbour wake-up. \\
\hline 4 & $\begin{array}{l}\text { Context-Aware Resource } \\
\text { Discovery [35] }\end{array}$ & $\begin{array}{l}\text { Learns higher and lower duty } \\
\text { cycles efficiently. }\end{array}$ & $\begin{array}{l}\text { Learning is available on static nodes, not } \\
\text { on mobile. }\end{array}$ \\
\hline 5 & Searchlight [35] & $\begin{array}{l}\text { Usage of probe node to discover } \\
\text { within shorter period. }\end{array}$ & Mobility agnostic method. \\
\hline 6 & Random Discovery [35] & Communication planning better. & More time wasted on data gathering. \\
\hline 7 & $\begin{array}{c}\text { Efficient Application-layer } \\
\text { Discovery Protocol (EADP) [35] }\end{array}$ & High discovery rate. & Time spent on node advertisement. \\
\hline 8 & Random Walk [38] & Easy and random search. & $\begin{array}{l}\text { Possibly back to the previous visited } \\
\text { node. }\end{array}$ \\
\hline 9 & $\begin{array}{l}\text { Restricted Random Walk (RRW) } \\
\text { [38] }\end{array}$ & $\begin{array}{l}\text { Random selection an adjacent } \\
\text { node never visited before. }\end{array}$ & $\begin{array}{l}\text { Non-computer network search will be } \\
\text { done random and recursively. }\end{array}$ \\
\hline 10 & $\begin{array}{l}\text { Breadth-First Search } \\
\text { (BFS) [38] }\end{array}$ & $\begin{array}{c}\text { Forwarding queries to all adjacent } \\
\text { nodes. }\end{array}$ & $\begin{array}{l}\text { A large number of queries inside the } \\
\text { network. }\end{array}$ \\
\hline 11 & Intelligent BFS (Int-BFS) [38] & Not flooding the entire network. & $\begin{array}{l}\text { Node selection based on how many } \\
\text { times the answering the queries. }\end{array}$ \\
\hline
\end{tabular}




\begin{tabular}{|c|c|c|c|}
\hline 12 & $\begin{array}{l}\text { Depth-First Search } \\
\text { (DFS) [38] }\end{array}$ & $\begin{array}{l}\text { Only forwards one query to one } \\
\text { adjacent node. }\end{array}$ & $\begin{array}{l}\text { More time to forward query until no } \\
\text { more not visited adjacent node. }\end{array}$ \\
\hline 13 & $\begin{array}{l}\text { Adaptive Probabilistic Search } \\
\text { (APS) [38] }\end{array}$ & $\begin{array}{l}\text { Searching capabilities improve } \\
\text { over time. }\end{array}$ & $\begin{array}{l}\text { Nodes have random and equal } \\
\text { probability to be selected. }\end{array}$ \\
\hline 14 & $\begin{array}{l}\text { Blackboard Resource Discovery } \\
\text { Mechanisms (BRDM) [38] }\end{array}$ & Artificial intelligence technique. & $\begin{array}{l}\text { Query will forward randomly if no } \\
\text { recommended node found. }\end{array}$ \\
\hline 15 & $\begin{array}{c}\text { Learning Automata-based } \\
\text { Resource Discovery (LARD) } \\
{[36]}\end{array}$ & $\begin{array}{l}\text { Simplify the problem of network } \\
\text { flooding. }\end{array}$ & Issue of fault positive error. \\
\hline 16 & $\begin{array}{c}\text { Improved Adaptive Probabilistic } \\
\text { Search (IAPS) [36] }\end{array}$ & $\begin{array}{l}\text { Better performance than popular } \\
\text { random walk/query and APS. }\end{array}$ & $\begin{array}{l}\text { Depend on the characteristics of ant- } \\
\text { colony optimisation. }\end{array}$ \\
\hline 17 & $\begin{array}{l}\text { Discovery of Heterogeneous } \\
\text { Multiple Compute Resources } \\
\text { Framework (DHMCF) [36] }\end{array}$ & Responds to dynamic requests. & Low robustness and security. \\
\hline 18 & $\begin{array}{l}\text { Content-Based Search } \\
\text { Techniques [37] }\end{array}$ & $\begin{array}{l}\text { Allow access to past and real-time } \\
\text { data and accurate search results. }\end{array}$ & High level results cannot be achieved. \\
\hline 19 & $\begin{array}{l}\text { Context-Based Search } \\
\text { Techniques [37] }\end{array}$ & $\begin{array}{l}\text { Efficient control to manage IoT } \\
\text { objects. }\end{array}$ & Difficult to acquire context-information. \\
\hline 20 & $\begin{array}{c}\text { Location-Based Search } \\
\text { Techniques [37] }\end{array}$ & $\begin{array}{l}\text { Coupled with query routing } \\
\text { techniques. }\end{array}$ & $\begin{array}{l}\text { Take more time and requires large } \\
\text { storage space. }\end{array}$ \\
\hline 21 & $\begin{array}{l}\text { Social structure-based search } \\
\text { techniques [37] }\end{array}$ & $\begin{array}{l}\text { More user behaviour and formed } \\
\text { by humans. }\end{array}$ & Traffic congestion and scalability issues. \\
\hline 22 & $\begin{array}{l}\text { Semantic and ontology-based } \\
\text { search techniques [37] }\end{array}$ & $\begin{array}{l}\text { Results can be modelled in } \\
\text { natural language. }\end{array}$ & $\begin{array}{l}\text { Requires integration of middleware for } \\
\text { management. }\end{array}$ \\
\hline 23 & $\begin{array}{c}\text { Resource and service discovery } \\
{[37]}\end{array}$ & Support QoS parameter. & $\begin{array}{l}\text { Requires additional subscription } \\
\text { management. }\end{array}$ \\
\hline
\end{tabular}

In the digital age, media is numeric data also. Ones and zeros represent it as digital data. It can be concluded that almost all techniques can be used to measure and tackle several parameters such as performance, latency, bandwidth, responsiveness, security, speed, robustness, usability, reliability, capacity, functionality, energy consumption, data integration and so on. We see there are two prominent techniques in current IIoT data searching, Context-Aware Resource Discovery and Context-Based Search Techniques. Context-Aware Resource Discovery under Learning Based Approach offer Q-learning as intended by [35] works by verifying an action-value function based on the interactions of the controller with the environment [39]. Q-learning is the latest Context-Aware Resource Discovery framework to make node discovery. Application of a model-free Qlearning algorithm to estimate optimal controller settings for time-invariant linear systems without any knowledge of the system dynamics. This Q-learning algorithm is a potential solution to address changing environments and configurations of IoT. Q-learning is one of the seen effective techniques available for IoT node discovery. The challenges of context-based have already been identified, and we have simplified it as matters of dedicated server management, data acquisition of IoT objects issues, and specific application requirements. Also, there are some apparent weaknesses as per view in Table 2, so implementation of this new technique still has significant reasons to be studied to enable in-depth research in future.

\section{SIMULATORS SELECTION \& RESULTS}

Because several network simulators have been used for IoT researches, however only three categories of simulators that can be used intensively based on scope and level of architectural layer coverage [40]. Comparison in Table 3 shows the shortlisted simulators that have updated and released in 2016 and above. All of the simulator categories are suitable for networking and IoT simulation, among others, have been developed in response to the emergence of the IoT paradigm, aiming to provide end-to-end support of all IoT elements. It also focuses on the aspects of large data processing IoT applications and to support the IoT paradigm, and then continue to expand to support the required IoT implementation. To verify the suitability of the simulator, and to avoid compatibility issues on future, seven related tools with different and varieties background involved are CubCarcon, COOja, OMNet++, Qualnet, iFogSim, IOTSim, and NS-3.

CupCarbon is also known as Smart City and Internet of Things Wireless Sensor Network (SCI-WSN) simulator. It offers two simulation environments; first to enables the design of mobility scenarios and the generation of natural events such as fires, gas, vehicles and flying objects, and second, to represents a discrete event simulation of wireless sensor networks [41]. It allows processes to configure each sensor's node individually and make program code generation for the network more convenient and algorithmic.

COOJA is a unique simulator to permit the simulation of real hardware platforms using the application of Contiki OS. It is capable of simulating wireless sensor network with cross-layer Java-based wireless sensor network simulator distributed with Contiki [42]. Contiki offers for the native platform as a shared library and then load the library into Java using Java Native Interfaces (JNI). This simulator allows to produce simulations at different levels ranging from physical layers to application layers, and also allows 
the simulations for hardware such as control system and sensor nodes.

OMNeT++ is a comprehensive simulator for researchers and academicians in diverse areas come with an extensible, modular, component-based $\mathrm{C}++$ simulation library and framework. It offers an Eclipse-based IDE, a graphical runtime environment, and a host of other tools. Furthermore, there are enhancement version of OMNet++ for real-time simulations, network simulation, database integration, SystemC integration, and some other functions [43]. Furthermore, the introduction of the INET Framework for use by OMNeT++ team is attractive to the community.

Qualnet is located in the category of commercial simulators. It can design and analyse the network performance and network protocols in 3D visualisation. Additionally, enhanced features like faster simulation speeds, scalability, advanced GUI support, excellent debugging support and parallel implementations, have had a significant impact on the research field. Researchers and developers today are increasingly keen to provide solutions to complex network problems, including IoT [44].

iFogSim is an open source simulator for critical areas in the cloud, edge computing, fog computing and IoT [45]. Although iFogSim is quite new and the emergence of an advanced version like MyiFogSim seems to make this simulator less attention, but its great function should be looking seriously. It enables the simulation process of fog computing and IoT environment to assess the level of resource management and policy scheduling across edge and cloud resources.

IOTSim is an academic project by group research and development by Newcastle University from United Kingdom collaboration with Australian National University and the University of Tasmania from Australia [46]. They were developed IOTSim collaboratively to achieve modelling of heterogeneous IoT device types, device virtualisation and resource management technologies, data programming abstractions, and data flows.

NS-3 is a free software simulator licensed under the GNU GPLv2 recommended for research and educational use and support for discrete-event network simulation [47]. The NS3 software basic infrastructures are very helpful to be used as a real-time network simulator, which is due to its realworld interconnectedness and enabling much real-world protocol execution. NS-3 can also support research on both IP and non-IP based and look more realistic with the facilitation of some use cases for interacting with real systems.

Within any big scale organisation or project, numerous devices are connected through sophisticated wireless technology for different applications such as communication, surveillance, security, message, social media, or even national defence. The emergence of IoT is particularly helpful to high-tech wireless equipment because of its ability to support massive IoT nor high-volume IoT. These high-volume IoT spreads are due to different paradigms including the Internet of Things (IoT), fog computing, edge and cloud computing. All of this dissemination, which requires an effective and highperformance network environment and conscious communication, have opened up simulation research areas.
Summarily, only four of them are supports the large scale and high-volume IoT. We look forward to implementing the NS-3 simulator because it has better credibility. NS-3 is based on an earlier version, NS-2. It can be used to create WSN simulations to replicate the IoT perception layer. $\mathrm{OMNeT}++$ have the same capability and similar features for related protocols, but NS-3 can reduce support for application-level protocols to make it simpler. As an opensource tool, NS-3 is a commercial product that can also be used in academic and IoT research widely

Table 3a: Comparison of IoT simulators (3 main features)

\begin{tabular}{|l|l|l|l|}
\hline Simulator & Volume & Support & Language \\
\hline $\begin{array}{l}\text { CupCarbon } \\
{[48]}\end{array}$ & Low-Volume & Very Good & $\begin{array}{l}\text { Java, } \\
\text { Custom }\end{array}$ \\
\hline $\begin{array}{l}\text { Cooja } \\
{[49]}\end{array}$ & Low-Volume & Very Good & Java, C \\
\hline $\begin{array}{l}\text { OMNet++ } \\
{[50]}\end{array}$ & High-Volume & Excellent & C++ \\
\hline $\begin{array}{l}\text { QualNet } \\
{[51]}\end{array}$ & High-Volume & Excellent & C++ \\
\hline $\begin{array}{l}\text { iFogSim } \\
{[52]}\end{array}$ & Low-Volume & Good & Java \\
\hline $\begin{array}{l}\text { IOTSim } \\
{[53]}\end{array}$ & High-Volume & Very Good & Java \\
\hline $\begin{array}{l}\text { NS-3 } \\
{[54]}\end{array}$ & High-Volume & Excellent & C++ \\
\hline
\end{tabular}

Table 3b: Comparison of IoT simulators (more 3 extra features)

\begin{tabular}{|l|l|l|l|}
\hline Simulator & $\begin{array}{l}\text { Level of } \\
\text { Acceptance }\end{array}$ & Mobility & $\begin{array}{l}\text { IoT } \\
\text { Standard }\end{array}$ \\
\hline $\begin{array}{l}\text { CupCarbon } \\
{[48]}\end{array}$ & Very High & Yes & $\begin{array}{l}\text { LoRa } \\
\text { WAN, } \\
\text { WSN, } \\
\text { ZigBee, } \\
802.15 .4\end{array}$ \\
\hline $\begin{array}{l}\text { Cooja } \\
{[49]}\end{array}$ & High & Yes & $\begin{array}{l}\text { All } \\
\text { protocols }\end{array}$ \\
\hline $\begin{array}{l}\text { OMNet++ } \\
\text { [50] }\end{array}$ & Very High & Yes & Manual \\
\hline $\begin{array}{l}\text { QualNet } \\
{[51]}\end{array}$ & Mod & Yes & ZigBee \\
\hline $\begin{array}{l}\text { iFogSim } \\
\text { [52] }\end{array}$ & High & No & No \\
\hline $\begin{array}{l}\text { IOTSim } \\
{[53]}\end{array}$ & High & No & No \\
\hline $\begin{array}{l}\text { NS-3 } \\
\text { [54] }\end{array}$ & Very High & Yes & LoRa WAN \\
\hline
\end{tabular}

\section{CONCLUSION}

In the future IoT environment, Q-learning is the fastest resource discovery in the ubiquitous network. It is the best discovery technique to take many portable IoT devices operated in a large-scale network. In a LoRa network, the device transmits data packets according to an uplink path, so, we could reduce the transmission delay by optimising the uplink performance. In this paper, we reviewed the 
selected papers and then chose the best simulator for verifying the IoT resource discovery in the LoRa network. NS-3 as a simulation tool that will produce better performance topologies using several numbers of experiments.

\section{ACKNOWLEDGMENT}

We fully acknowledged TATI University College and Universiti Sultan Zainal Abidin as our employer and sponsor. This research publication is funded by UniSZA research grant (UniSZA/2017/DPU/73).

\section{REFERENCES}

1. Y. Ma, J. Rao, W. Hu, X. Meng, X. Han, Y. Zhang, Y. Chai, and C. Liu, "An efficient index for massive IoT data in cloud environment," 21st ACM International Conference on Information and Knowledge Management, 2012, pp. 2129-2133.

2. M. Chen, Y. Miao, Y. Hao, and K. Hwang, "Narrow band Internet of Things," IEEE Access, 5, 2017, pp. 2169-3536.

3. J. Xu, J. Yao, L. Wang, Z. Ming, K. Wu, and L. Chen, "Narrowband Internet of Things: Evolutions, technologies, and open issues," IEEE Internet of Things Journal, 5(3), 2018, pp. 1449-1462.

4. A. Banafa, "Three major challenges facing IoT," IEEE Internet of Things Journal, 2(3), 2017, pp. 1449-1462.

5. T. L. Koreshoff, T. Robertson, and T. W. Leong, "Internet of Things: A review of literature and products," ACM 25th Australian Computer-Human Interaction Conference: Augmentation, Application, Innovation, Collaboration, 2013, pp. 335-344.

6. M. S. Satya, "Edge computing: Vision and challenges," IEEE Internet of Things Journal, 3(5), 2016, pp. 637646.

7. F. Malandrino, S. Kirkpatrick, and C. F. Chiasserini, "How close to the edge? Delay/utilization trends in MEC," ACM Workshop on Cloud-Assisted Networking, 2016, pp. 37-42.

8. T. F. Rahman, and C. Sacchi, "A cooperative radio resource management strategy for mobile multimedia LTE uplink," IEEE Aerospace Conference, 2014, pp. 18.

9. M. R. Mardani, S. Mohebi, and H. Bobarshad, "Robust uplink resource allocation in LTE networks with M2M devices as an infrastructure of Internet of Things," IEEE 4th International Conference on Future Internet of Things and Cloud, 2016, pp. 186-193.

10. S. H. da Mata, and P. R. Guardieiro, "A genetic algorithm based approach for resource allocation in LTE uplink," IEEE International Telecommunications Symposium, 2014, pp. 1-5.

11. T. Hossain, Y. Doi, T. Tazin, M. A. R. Ahad, and S. Inoue, "Study of LoRaWAN technology for activity recognition," ACM International Joint Conference and International Symposium on Pervasive and Ubiquitous Computing and Wearable Computers, 2018, pp. 14491453.

12. Rahman, Arrief, and Muhammad Suryanegara. "The development of IoT LoRa: A performance evaluation on LoS and Non-LoS environment at $915 \mathrm{MHz}$ ISM frequency," IEEE International Conference on Signals and Systems, 2017, pp. 163-167.

13. H. Hejazi, H. Rajab, T. Cinkler, and L. Lengyel, "Survey of platforms for massive IoT," IEEE International Conference on Future IoT Technologies, 2018, pp. 1-8.

14. V. M. Suresh, R. Sidhu, P. Karkare, A. Patil, Z. Lei, and A. Basu, "Powering the IoT through embedded machine learning and LoRa," IEEE 4th World Forum on Internet of Things, 2018, pp. 349-354.

15. H. C. Lee, and K. H. Ke, "Monitoring of large-area IoT sensors using a LoRa wireless mesh network system: Design and evaluation," IEEE Transactions on Instrumentation and Measurement, 67(9), 2018, pp. 2177-2187.

16. A. Lavric, and V. Popa, "Internet of Things and LoRaTM low-power wide-area networks: A survey," IEEE International Symposium on Signals, Circuits and Systems, 2017, pp. 1-5.

17. H. Rudeš, I. N. Kosović, T. Perković, and M. Čagalj, "Towards reliable IoT: Testing LoRa communication," IEEE 26th International Conference on Software, Telecommunications and Computer Networks, 2018, pp. 1-3.

18. I. Butun, N. Pereira, and M. Gidlund, "Analysis of LoRaWAN v1. 1 security," 4th ACM MobiHoc Workshop on Experiences with the Design and Implementation of Smart Objects, 2018, pp. 1-6.

19. B. Reynders, Q. Wang, and S. Pollin, "A LoRaWAN module for ns-3: Implementation and evaluation," ACM 10th Workshop on ns-3, 2018, pp. 61-68

20. A. Rahmadhani, and F. Kuipers, "When LoRaWAN frames collide," 12th International Workshop on Wireless Network Testbeds, Experimental Evaluation and Characterization, 2018, pp. 1-9.

21. M. O. Farooq, and D. Pesch, "Analyzing LoRa: A use case perspective," IEEE 4th World Forum on Internet of Things, 2018, pp. 355-360.

22. Y. Ma, J. Rao, W. Hu, X. Meng, X. Han, Y. Zhang, Y. Chai, and C. Liu, "An efficient index for massive IoT data in cloud environment," 21st ACM International Conference on Information and Knowledge Management, 2012, pp. 2129-2133.

23. G. Bloom, B. Alsulami, E. Nwafor, and I. Cibrario Bertolotti, "Design patterns for the industrial Internet of Things," 14th IEEE International Workshop on Factory Communication Systems, 2018, pp. 1-10.

24. K. A. Delic, "On resilience of IoT systems: The Internet of Things (ubiquity symposium)," Ubiquity, 2016(no. February), 2016, pp. 1-7.

25. S. Egelman, R. Kannavara, and R. Chow, "Is this thing on? Crowdsourcing privacy indicators for ubiquitous sensing platforms," 33rd Annual ACM Conference on Human Factors in Computing Systems, 2015, pp. 16691678.

26. M. H. Syed, E. B. Fernandez, and M. Ilyas, "A pattern for fog computing," ACM 10th Travelling Conference on Pattern Languages of Programs, 2016, pp. 1-10.

27. M. Ketel, "Fog-cloud services for IoT," ACM SouthEast Conference, 2017, pp. 262-264.

28. S. Yi, C. Li, and Q. Li, "A survey of fog computing: Concepts, applications and issues," ACM 2015 Workshop on Mobile Big Data, 2015, pp. 37-42.

29. F. Bonomi, R. Milito, J. Zhu, and S. Addepalli, "Fog computing and its role in the Internet of Things," ACM 1st MCC Workshop on Mobile Cloud Computing, 2012, pp. 13-16.

30. Y. Xue, J. Li, S. Nazarian, and P. Bogdan, "Fundamental challenges toward making the IoT a reachable reality: A model-centric investigation," ACM Transactions on Design Automation of Electronic Systems, 22(3), 2017, pp. 1-25. 
31. Y. Shi, G. Ding, H. Wang, H. E. Roman, and S. Lu, "The fog computing service for healthcare," IEEE 2nd International Symposium on Future Information and Communication Technologies for Ubiquitous HealthCare, 2015, pp. 1-5.

32. G. D. F. Morales, A. Bifet, L. Khan, J. Gama, and W. Fan, "IoT big data stream mining," 22nd ACM SIGKDD International Conference on Knowledge Discovery and Data Mining, 2016, pp. 2119-2120.

33. P. Barnaghi, and A. Sheth, "On searching the Internet of Things: Requirements and challenges," IEEE Intelligent Systems, 31(6), 2016, pp. 71-75.

34. Y. Fathy, P. Barnaghi, and R. Tafazolli, "Large-scale indexing, discovery, and ranking for the Internet of Things (IoT)," ACM Computing Surveys, 51(2), 2018, pp. 1-53.

35. M. L. Valarmathi, L. Sumathi, and G. Deepika, "A survey on node discovery in Mobile Internet of Things (IoT) scenarios," IEEE 3rd International Conference on Advanced Computing and Communication Systems, 2016, pp. 1-5.

36. A. A. Jamal, and W. J. Teahan, "Alpha multipliers breadth-first search technique for resource discovery in unstructured peer-to-peer networks," International Journal on Advanced Science, Engineering and Information Technology, 7(4), 2017, pp. 1403-1412.

37. S. Pattar, R. Buyya, K. R. Venugopal, S. S. Iyengar, and L. M. Patnaik, "Searching for the IoT resources: Fundamentals, requirements, comprehensive review, and future directions." IEEE Communications Surveys and Tutorials, 20(3), 2018, pp. 2101-2132.

38. A. A. Jamal, W. S. W. Awang, M. F. A. Kadir, A. A. Aziz, and W. J. Teahan, "Implementation of resource discovery mechanisms onto PeerSim," 3rd International Conference on Informatics and Applications, 2014, pp. 51-55.

39. A. Farahmandi, G. A. Hewer, B. C. Reitz, K. Estabridis, and K. G. Vamvoudakis, "A model free learning algorithm to control autonomous streams over IoT," 8th International Conference on the Internet of Things, 2018, pp. 1-4.

40. M. Chernyshev, Z. Baig, O. Bello, and S. Zeadally, "Internet of Things (IoT): research, simulators, and testbeds," IEEE Internet of Things Journal, 5(3), 2018 , pp. 1637-1647.

41. CubCarbon, Home. 2018, Available: http://www.cupcarbon.com/.

42. Cooja, An Introduction to Cooja. 2018, Available: https://github.com/contiki-os/contiki/wiki/An-

Introduction-to-Cooja.

43. OpenSim Ltd., OMNet++. 2018, Available: https://omnetpp.org.

44. QualNet, Qualnet Simulator. 2018, Available: http:// http://phdprojects.org/qualnet-simulator/.

45. iFogSim, iFogSim: An Open Source Simulator for Edge Computing, Fog Computing and IoT. 2018, Available: https://opensourceforu.com/2018/12/ifogsim-an-opensource-simulator-for-edge-computing-fog-computingand-iot/.

46. IOTSim, IOTSim: Modeling and Simulation in the IoT and Big Data Era. 2018, Available: https://rajivranjan.net/iotsim/.

47. nsnam, NS-3: Network Simulator. 2018, Available: https://www.nsnam.org/.

48. A. Bounceur, O. Marc, M. Lounis, J. Soler, L. Clavier, P. Combeau, R. Vauzelle, L. Lagadec, R. Euler, M. Bezoui, and P. Manzoni, "Cupcarbon-lab: An IoT emulator," 15th IEEE Annual Consumer Communications \& Networking Conference, 2018, pp. 1-2.

49. A. H. Patil, N. Goveas, and K. Rangarajan, "Rearchitecture of Contiki and Cooja regression test suites using combinatorial testing approach," ACM SIGSOFT Software Engineering Notes, 40(2), 2015, pp. 1-3.

50. V. Inzillo, A. F. Santamaria, and A. A. Quintana, "Integration of Omnet++ simulator with MATLAB for realizing an adaptive beamforming system," IEEE/ACM 21st International Symposium on Distributed Simulation and Real Time Applications, 2017, pp. 1-2.

51. P. Kumar, M. Ruthwik, M. K. Sree, M. Saad, and A. P. Patil, "Implementation of an emergency evacuation system using intelligent routing using QualNet Simulator," ACM Symposium on Women in Research, 2016, pp. 131-138.

52. M. M. Lopes, W. A. Higashino, M. A. M. Capretz, and L. F. Bittencourt, "Myifogsim: A simulator for virtual machine migration in fog computing," ACM 10th International Conference on Utility and Cloud Computing, 2017, pp. 47-52.

53. X. Zeng, S. K. Garg, P. Strazdins, P. P. Jayaraman, D. Georgakopoulos, and R. Ranjan, "IOTSim: A simulator for analysing IoT applications," Journal of Systems Architecture, 72, 2017, pp. 93-107.

54. K. Nagori, M. Balachandran, A. Deepak, M. P. Tahiliani, and B. R. Chandavarkar, "Common TCP evaluation suite for ns-3: Design, implementation and open issues," ACM Workshop on ns-3, 2017, pp. 9-16. 